\title{
Noonan Syndrome in South Africa: Clinical and Molecular Profiles
}

\begin{abstract}
Cedrik Tekendo-Ngongang ${ }^{1 \dagger}$, Gloudi Agenbag ${ }^{1}$, Christian Domilongo Bope ${ }^{1,2}$, Alina Izabela Esterhuizen ${ }^{1,3}$ and Ambroise Wonkam ${ }^{1,4 *}$

${ }^{1}$ Division of Human Genetics, Departments of Medicine and Pathology, Faculty of Health Sciences, University of Cape Town, Cape Town, South Africa, ${ }^{2}$ Departments of Mathematics and Computer Sciences, Faculty of Sciences, University of Kinshasa, Kinshasa, Democratic Republic of Congo, ${ }^{3}$ National Health Laboratory Service, Groote Schuur Hospital, Cape Town, South Africa, ${ }^{4}$ Faculty of Health Sciences, Institute of Infectious Diseases and Molecular Medicine, University of Cape Town, Cape Town, South Africa
\end{abstract}

Noonan Syndrome (NS) is a common autosomal dominant multisystem disorder, caused by mutations in more than 10 genes in the Ras/MAPK signaling pathway. Differential mutation frequencies are observed across populations. Clinical expressions of NS are highly variable and include short stature, distinctive craniofacial dysmorphism, cardiovascular abnormalities, and developmental delay. Little is known about phenotypic specificities and molecular characteristics of NS in Africa. The present study has investigated patients with NS in Cape Town (South Africa). Clinical features were carefully documented in a total of 26 patients. Targeted Next-Generation Sequencing (NGS) was performed on 16 unrelated probands, using a multigene panel comprising 14 genes: PTPN11, SOS1, RIT1, A2ML1, BRAF, CBL, HRAS, KRAS, MAP2K1, MAP2K2, NRAS, RAF1, SHOC2, and SPRED1. The median age at diagnosis was 4.5 years (range: 1 month-51 years). Individuals of mixed-race ancestry were most represented (53.8\%), followed by black Africans (30.8\%). Our cohort revealed a lower frequency of pulmonary valve stenosis (34.6\%) and a less severe developmental milestones phenotype. Molecular analysis found variants predicted to be pathogenic in $5 / 16$ cases (31.2\%). Among these mutations, two were previously reported: MAP2K1-c.389A>G (p.Tyr130Cys) and PTPN11 - c.1510A>G (p.Met504Val); three are novel: CBL-c.2520T>G (p.Cys840Trp), PTPN11- c.1496C > T (p.Ser499Phe), and MAP2K1 - c.200A > C (p.Asp67Ala). Molecular dynamic simulations indicated that novel variants identified impact the stability and flexibility of their corresponding proteins. Genotype-phenotype correlations showed that clinical features of NS were more typical in patients with variants in MAP2K1. This first application of targeted NGS for the molecular diagnosis of NS in South Africans suggests that, while there is no major phenotypic difference compared to other populations, the distribution of genetic variants in NS in South Africans may be different.

Keywords: Noonan syndrome, multigene panel testing, targeted next-generation sequencing, RASopathies, Ras/MAPK signaling pathway, South Africa

\section{INTRODUCTION}

Noonan syndrome (NS; MIM 163950) is a common autosomal dominant condition, with an estimated global incidence of 1:1,000 to 1:2,500 live births (Mendez and Opitz, 1985). Affected individuals present with multisystem involvement, including short stature, distinctive craniofacial dysmorphism, congenital heart defects (CHD), skeletal abnormalities, developmental delay, 
coagulation defects, and other abnormalities (Allanson and Roberts, 2016). Noonan syndrome is clinically heterogeneous with significant interfamilial and intrafamilial variable expression. Noonan syndrome condition is caused by heterozygous germline mutations in more than 10 genes encoding, either proteins of the Ras family of GTPases (KRAS, NRAS, RIT1, and RRAS), or modulators of Ras function (PTPN11, SOS1, SOS2, CBL, RASA2, and SHOC2) and downstream signal transducers (RAF1, BRAF, and MAP2K1) (Cordeddu et al., 2015; Aoki et al., 2016). To date, mutations in all identified genes for NS result in gain-of-function within the Ras/MAPK pathway, and account for up to $80 \%$ of NS cases (Aoki et al., 2016). In many clinical settings, missense variants in PTPN11 alone are found in about $50 \%$ of affected individuals (Tartaglia and Gelb, 2005), while SOS1 has been reported to be the second most mutated gene, accounting for $10-20 \%$ of PTPN11-negative patients (Roberts et al., 2007). Compared to PTPN11 and SOS1, the contribution of other known genes seems to be minimal, with variable mutation frequencies observed across populations (Allanson and Roberts, 2016). Recent studies have reported association between biallelic variants in LZTR1 and NS phenotype (Johnston et al., 2018; Nakaguma et al., 2019), supporting the existence of an autosomal recessive form of the condition, as suggested by some clinical studies (Abdel-Salam and Temtamy, 1969; Maximilian et al., 1992; Van Der Burgt and Brunner, 2000). However, heterozygous variants in LZTR1 have also been previously associated to the NS phenotype in at least seven families (Chen et al., 2014; Yamamoto et al., 2015), and the consequences of LZTR1 pathogenic variants on the Ras/MAPK signaling pathway remain to be clarified. The clinical diagnosis of NS is often straightforward in several cases, through recognition of key craniofacial and musculoskeletal features, in combination with CHD (Romano et al., 2010). Phenotypic overlap with other conditions sharing the same pathogenetic mechanisms so-called RASopathies, challenges the diagnosis of NS in some patients and families; this is particularly true for Cardiofaciocutaneous syndrome (CFC) and Costello syndrome. As evidenced by several genetic studies, phenotypic expressivity in many conditions may show variations across populations and ethnic groups, making it more challenging to apply well-established clinical diagnostic criteria universally (Tekendo-Ngongang et al., 2014; Kruszka et al., 2017). Affected individuals with NS from sub-Saharan Africa have seldom been reported in the literature (Kruszka et al., 2017). Furthermore, no study investigating the genetic etiology of NS in South Africans has been previously conducted, despite that numerous individuals and families affected with NS were identified in South Africa, largely due to unavailability of molecular diagnostic testing for RASopathies in the state public sector. The present study aimed at characterizing a cohort of South African patients with NS from clinical and molecular perspectives, using targeted NGS approach.

\section{MATERIALS AND METHODS}

\section{Ethical Approval}

The study was performed in accordance with the Declaration of Helsinki and with the approval of the Faculty of Health Sciences
Human Research Ethics Committee, University of Cape Town (HREC: 449/2016). Written informed consent was obtained from the parents and/or the patient prior to their involvement into the study, including permission to publish photographs.

\section{Patients and Phenotyping}

This study was conducted in Cape Town, South Africa, and included 26 participants (20 children and six adults); among them, 20 were unrelated. Patients were recruited through the University of Cape Town (UCT) affiliated Hospitals, namely Red Cross War Memorial Children's Hospital (RCWMCH) and Groote Schuur Hospital (GSH). Patients were selected retrospectively and prospectively using the Van der Burgt scoring system for clinical diagnosis of NS (Van der Burgt, 2007). All pediatric and adult patients were assessed by trained clinical geneticists familiar with RASopathies. For each proband, family history suggestive of NS was systematically assessed by means of three or more generations pedigree. Phenotypic data recorded included all clinical characteristics, with emphasis on antenatal features, NS-specific dysmorphology assessment, investigation of bleeding diathesis and cardiovascular abnormalities on Electrocardiograms (ECG) and echocardiograms.

\section{Molecular Methods}

Genomic DNA of each selected patient was isolated from peripheral blood leukocytes at the National Health Laboratory Service (NHLS)-Molecular Genetics Laboratory, GSH, following the manufacturer's instructions, the standard Maxwell 16 protocol (Maxwell ${ }^{\circledR} 16$ Blood DNA purification kit, Promega, Madison, WI 53711, USA).

\section{Targeted Gene Panel Sequencing and Variants Analysis}

Of the 26 DNA samples isolated, 16 DNA samples from unrelated patients were genotyped. Targeted Sequencing was performed with the Ion Torrent platform, at the sequencing laboratory of the Division of Human Genetics of UCT, using the Ion PGM ${ }^{\mathrm{TM}}$ system (Thermo Fisher Scientific, Waltham, Massachusetts, USA). Pre-designed primers for Ion AmpliSeq Noonan Research Panel were used (Life Technologies, Carlsbad, CA). The primers amplify exons and intron/exon boundaries of 14 genes known to be associated with NS and related conditions, including A2ML1, BRAF, CBL, HRAS, KRAS, MAP2K1, MAP2K2, NRAS, PTPN11, RAF1, RIT1, SHOC2, SOS1, and SPRED1. This multigene panel is predicted to cover $100 \%$ of the targeted regions, in 268 amplicons (Nelen et al., 2014). Sequencing data analysis, including quality assessment, read alignment, variants identification, variant annotation, visualization, and prioritization was primarily performed using the bioinformatics pipeline of the Ion Torrent Suite and the Ion Reporter cloud-based software (Thermo Fisher Scientific, Waltham, Massachusetts, USA). From the usable reads, $99 \%$ could be mapped to the human reference genome used (Homo sapiens, hg19, build 37.2). Further manual analysis was executed for variant prioritization and interpretation based on the variant call format (VCF) file generated by the Ion Reporter software. In this step, variants were prioritized using their minor allele frequency $(\mathrm{MAF}<0.01)$ based on 
1,000 genomes and 5,000 exomes projects, their zygosity, their function, their location within the gene, and their pathogenicity according to ClinVar. A parallel analysis of sequencing data was performed based on the binary alignment map (BAM) file generated by the Ion Reporter software. Picard package with option to SortSAM, MarkDuplicates and FixMateInformation on a per-sample basis were used to sort coordinate, mark polymerase chain reaction (PCR) duplicate reads and verify mate-pair information, respectively (Mckenna et al., 2010). The variant calling was done using Samtools, bcftools (Li et al., 2009) and Varscan 2 (Koboldt et al., 2012) with the reference Human genome (Hg19; build 37.2). The conservation and deleteriousness of the variant were investigated using ANNOVAR which interrogated the following tools: SIFT, PolyPhen 2 HVAR, Polyphen2 HDIV, MutationTaster, MutationAssessor, Likelihood ratio test (LRT), FATHMM, MetaSVM, MetaLR, GERP++, PhyloP, VEST3, DANN, CADD, PROVEAN, Fathmm-MKL, Integrated_fitCons, SiPhy_29way, PhastCons (Wang et al., 2010). The second level of variant filtration to avoid false positives or false negatives was conducted on annotated VCF files using an inhouse python script to select and retain only deleterious diseasecausing variants that have functional prediction using the 19 tools interrogated by ANNOVAR. The in-house python script uses two approaches to select deleterious variant (i) free hypothesis: cast of the vote of the annotated variant filter for "Deleterious or damaging disease-causing (D)" among annotation prediction tools based on a defined cut-off ( $\sim 50 \%)$; (ii) non free hypothesis: provide a list of known genes of the study with another level of prediction cut-off ( $25 \%$ ) (Lebeko et al., 2017). The cut-off for both hypothesis is defined as follow; (i) free hypothesis: select only variant which 10 tools interrogated by ANNOVAR predicted presence of a "D"; (ii) no free hypothesis: select only the gene which 5 tools interrogated by ANNOVAR predicted presence of a " $D$ " using the list of 14 NS genes analyzed. Existing online databases for previously reported NS variants and published literature on NS-associated variants were consulted for each candidate variant.

\section{Sanger Sequencing Validation and Family Segregation Studies}

All variants identified and predicted to be pathogenic were subsequently confirmed with Sanger capillary direct cycle sequencing and capillary electrophoresis using standard protocols. Depending on availability of family members, segregation studies were performed: the proband's parents were screened to ascertain the origin of the variant. In addition, and where possible, other available family members clinically affected or not, were screened for the identified variant, using Sanger direct cycle sequencing.

\section{D Protein Structure Prediction for Functional Characterization of Novel Variants}

Molecular dynamic (MD) simulations were conducted to assess the effect of novel variants on proteins function. The tertiary structure of the PTPN11 protein SHP-2 (PDB id: 2SHP) (Hof et al., 1998) and the MAP2K1 structure (UniProKD: A4QPA9) were generated using I-tasser homology webserver (Zhang, 2008). The CBL structure was generated by combining (PDB id: $1 F B V)$ and homology model. Six independent MD simulations were conducted; (i) MAP2K1 mutant and wild-type (WT), SHP2 mutant and WT, and CBL mutant and WT. All MD simulations were conducted with the GROMACS package, version 4.6.5 (Pronk et al., 2013) using OPLS force field (Jorgensen and Tiradorives, 1988). The systems were simulated in cubic box and solvated in water TIP3P (Neira et al., 1996). The temperature and pressure were maintained at $300 \mathrm{~K}$ using the ParrinelloDonadio-Bussi V-rescale thermostat (Bussi et al., 2007) and a pressure of 1 bar using the Berendsen barostat (Berendsen et al., 1984). The short-range non-bonded interactions were modeled using Lennard Jones potentials. The long-range electrostatic interactions were calculated using the particle mesh Ewald (PME) algorithm (Darden et al., 1993; Essmann et al., 1995). The LINCS algorithm was used to constrain all bond lengths (Hess et al., 1997) and the velocities were assigned according to the MaxwellBoltzman distribution at $300 \mathrm{~K}$.

\section{RESULTS}

\section{Socio-Demographics}

A total of 26 patients were included in this study, mostly unrelated $(n=20 ; 77 \%)$. The majority were children $(n=$ $20 ; 77 \%)$ with a median age at clinical diagnosis of 4.5 years (range: 1 month-51 years). Our cohort had preponderance of individuals of mixed-ancestry background (53.8\%), followed by black Africans (30.8\%). There was a slight predominance of males (sex ratio: 1.3; 15 males: 11 females).

\section{Phenotypic Description}

Gross motor milestones were on par for most patients, with ability to walk before the age of 18 months in $61.5 \%$ ( $n=$ $16 / 26)$ of cases. Six $(6 / 26 ; 23.1 \%)$ patients were unable to walk after 24 months. Speech delay was reported in 50\% (13/26) of cases. Craniofacial features were widely variable, but more characteristic in infants (2-12 months; 4/26), with widely spaced eyes, epicanthic folds and ptosis found in $75 \%$ of cases (Table 1). Comparison of six key physical characteristics (short stature, ptosis, widely spaced eyes, epicanthic folds, low-set ears, and webbed neck) between ethnic groups showed that, features were less frequent in Caucasians (2/26). Black Africans (8/26) presented with the most consistent dysmorphic features, with epicanthic folds $(87.7 \%)$, ptosis (75\%), and low-set ears (75\%) found to be the most common features (Table 2). At least one cardiovascular abnormality was identified in $65.4 \%(17 / 26)$ of patients. The most common $\mathrm{CHD}$ was pulmonary valve stenosis (PS), found in 34.6\% (9/26) of our cohort. Hypertrophic Cardiomyopathy (HCM) was identified in 19.2\% (5/26) and left axis deviation on ECG was found in $23.1 \%(6 / 26)$ of cases. The complete list of clinical features identified in our cohort of 26 patients can be found in the Table S1. 
TABLE 1 | Comparison of craniofacial features between age groups in the cohort of 26 patients.

\begin{tabular}{|c|c|c|c|c|}
\hline Features & $\begin{array}{c}\text { New-born } \\
(n=1)\end{array}$ & $\begin{array}{l}2-12 \text { months } \\
\qquad(n=4)\end{array}$ & $\begin{array}{c}1-12 \text { years } \\
(n=15)\end{array}$ & $\begin{array}{c}>18 \text { years } \\
\quad(n=6)\end{array}$ \\
\hline Macrocephaly & $1(100 \%)$ & $2(50 \%)$ & 0 & 0 \\
\hline Tall and prominent forehead & $1(100 \%)$ & $2(50 \%)$ & $8(53.3 \%)$ & $1(16.7 \%)$ \\
\hline Coarse face & 0 & $1(25 \%)$ & $2(13.3 \%)$ & $5(83.3 \%)$ \\
\hline Elongated face & $1(100 \%)$ & $1(25 \%)$ & $6(40 \%)$ & $4(66.7 \%)$ \\
\hline Widely spaced eyes & 0 & $3(75 \%)$ & 5 (33.3) & 0 \\
\hline Epicanthic folds & $1(100 \%)$ & $3(75 \%)$ & $9(60 \%)$ & $3(50 \%)$ \\
\hline Ptosis & 0 & $3(75 \%)$ & $10(66.7 \%)$ & 0 \\
\hline Low-set ears & $1(100 \%)$ & $2(50 \%)$ & $9(60 \%)$ & $3(50 \%)$ \\
\hline $\begin{array}{l}\text { Short, broad, depressed } \\
\text { nasal root }\end{array}$ & 0 & $3(75 \%)$ & 12 (80\%) & $3(50 \%)$ \\
\hline Prominent naso-labial folds & 0 & 0 & $2(13.3 \%)$ & $2(33.3 \%)$ \\
\hline $\begin{array}{l}\text { High wide peaks of the } \\
\text { vermilion }\end{array}$ & 0 & $2(50 \%)$ & $11(73.3)$ & $2(33.3 \%)$ \\
\hline Short neck & 0 & $2(50 \%)$ & $8(53.3 \%)$ & $2(33.3 \%)$ \\
\hline Webbed neck & 0 & 1 (25\%) & 3 (20\%) & 1 (16.7\%) \\
\hline
\end{tabular}

TABLE 2 | Comparison of six key dysmorphic features between ethnic groups.

\begin{tabular}{lcccc}
\hline Features & $\begin{array}{c}\text { Black african } \\
(\boldsymbol{n}=\mathbf{8})\end{array}$ & $\begin{array}{c}\text { Mixed-race ancestry } \\
(\boldsymbol{n}=14)\end{array}$ & $\begin{array}{c}\text { Caucasian } \\
(\boldsymbol{n}=\mathbf{2})\end{array}$ & $\begin{array}{c}\text { Indian } \\
(\boldsymbol{n}=\mathbf{2})\end{array}$ \\
\hline Widely spaced & $2(25 \%)$ & $5(35.7 \%)$ & 0 & $1(50 \%)$ \\
eyes & $6(75 \%)$ & $7(50 \%)$ & 0 & 0 \\
Ptosis & $7(87.5 \%)$ & $9(64.2 \%)$ & 0 & $1(50 \%)$ \\
Epicanthic folds & $6(75 \%)$ & $7(50 \%)$ & $1(50 \%)$ & $1(50 \%)$ \\
Low-set ears & $3(37.5 \%)$ & $1(7 \%)$ & $1(50 \%)$ & 0 \\
Webbed neck & $7(87.5 \%)$ & $12(87.5 \%)$ & 0 & $2(100 \%)$ \\
Short stature & & & & \\
\hline
\end{tabular}

\section{Genetic Variants Profile}

Following targeted NGS of 16 DNA samples from unrelated patients, in silico predictive algorithms supported the classification of seven heterozygous missense variants as pathogenic (7/16; Table 3; Figure 1). The novel variants were $C B L$ c.2520T $>\mathrm{G}$ (p.Cys840Trp), PTPN11 c.1496C >T (p.Ser499Phe), and MAP2K1 c.200A>C (p.Asp67Ala). Analysis confirmed segregation of three novel variants with the disease in the respective families. Segregation analysis revealed a possible case of germline mosaicism in a family where the unaffected father does not carry the variant (MAP2K1; c.200A>C) found in his two affected children. These children were half-brothers, born from two separate mothers.

The two variants previously reported in ClinVar as pathogenic are MAP2K1 c.389A $>$ G (p.Tyr130Cys) and PTPN11 c.1510A $>\mathrm{G}$ (p.Met504Val). Two identified $C B L$ variants were predicted to be pathogenic by prediction tools, but were reported as benign: c. 1858C $>\mathrm{T}$ (p. p.Leu620Phe) and as variant of uncertain significance: c.2345C $>$ T (p.Pro782Leu) in ClinVar. Correlatively, $C B L$ c. $2345 \mathrm{C}>\mathrm{T}$ failed to segregate with the disease in a large dominant family (Figure S1).

\section{Molecular Dynamic (MD) Simulations for Novel Variants}

Molecular dynamic (MD) simulations (Figure 2) showed that: for CBL, substitution of the negatively charged and hydrophilic amino acid Cys840 with the non-polar and hydrophobic amino acid Trp840 could impact binding interactions, stability and the flexibility of the protein.

For MAP2K1, substitution of the negatively charged and hydrophilic amino acid Asp67 with the non-polar and hydrophobic amino acid Ala67 could impact binding interactions; for SHP-2, substitution of the polar and hydrophilic amino acid Ser499 with the non-polar and highly hydrophobic amino acid Phe499 in the active site of the protein could impact binding interactions and the stability of structure.

\section{Genotype-Phenotype Correlations}

Correlations between the five variants that were predicted to be pathogenic, and NS related phenotypes are presented in Table 4. Comparisons showed that patients with variants in MAP2K1 were diagnosed earlier (mean age at diagnosis: 1 year) and presented with more typical clinical features of NS, followed by patients with variants in PTPN11 (mean age at diagnosis: 3.3 years; Figure 3). The patient with a variant in $C B L$ was found to have more discreet clinical features (Table 5).

\section{DISCUSSION}

This study provides a unique insight into the clinical and molecular profiles of South African individuals affected by NS, a rare attempt to comprehensively describe this condition in Africa. Patients were diagnosed relatively late, which could be explained by at least three factors: Firstly, the diagnosis of NS was hardly hypothesized and explored by clinicians in prenatal settings, partly due to the absence of prenatal molecular diagnostic testing for RASopathies in South Africa. A prenatal detection rate of $17.3 \%$ was reported elsewhere (Croonen et al., 2013). Secondly, in South Africa and Africa at large, the scarceness of trained medical geneticists often results in initial misdiagnosis. Finally, age-related variability in NS physical features makes its clinical diagnosis less easy for some professionals: moderate-to-severely affected individuals would be expected to be diagnosed by childhood, and mildly affected individuals in adulthood following either cardiac decompensation or cascade screening after the birth of a severely affected child (Van der Burgt, 2007; Roberts et al., 2013). Indeed, in the present study, all adult patients were diagnosed with NS during their assessment in the cardiology department. The developmental motor milestone in this cohort of South African patients was comparable to that reported in the literature, with $61.5 \%$ of patients being able to walk by the age of 18 months (Sharland et al., 1992). Half (50\%) of our patients were able to speak simple two-word sentences before the age of 24 months, far below the average age (31 to 32 months) of simple twoword sentences in NS reported by other authors (Pierpont, 2016). Nevertheless, these differences may be considered with caution due to the small size of our cohort and the fact that only 
TABLE 3 | Characteristics of pathogenic variants identified.

\begin{tabular}{llllllllll}
\hline Locus & Transcript & Gene & Exon & $\begin{array}{l}\text { Nucleotide } \\
\text { substitution }\end{array}$ & $\begin{array}{l}\text { Amino acid } \\
\text { substitution }\end{array}$ & $\begin{array}{l}\text { Variant } \\
\text { function }\end{array}$ & $\begin{array}{l}\text { Family } \\
\text { status }\end{array}$ & ClinVar variation ID & Associated disease \\
\hline chr15:66729181 & NM_002755.3 & MAP2K1 & 3 & c.389A>G & p.Tyr130Cys & Missense & Unknown & 13351 & CFC \\
chr15:66727484 & NM_002755.3 & MAP2K1 & 2 & C.200A>C & p.Asp67Ala & Missense & Familial & Not in ClinVar & NS \\
chr11:119170290 & NM_005188.3 & CBL & 16 & c.2520T>G & p.Cys840Trp & Missense & Familial & Not in ClinVar & NS \\
chr12:112926890 & NM_002834.3 & PTPN11 & 13 & c.1510A>G & p.Met504Val & Missense & Unknown & 40562 & NS \\
chr12:112926876 & NM_002834.3 & PTPN11 & 13 & c.1496C>T & p.Ser499Phe & Missense & Familial & Not in ClinVar & NS
\end{tabular}

\section{A}

\section{MAP2K1 c.200A >C, p.Asp67Ala}

\section{B}

PTPN11 c.1496C > T, p.Ser499Phe
C

CBL c. $2520 \mathrm{~T}>\mathrm{G}$, p.Cys840Trp

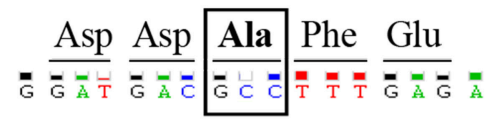

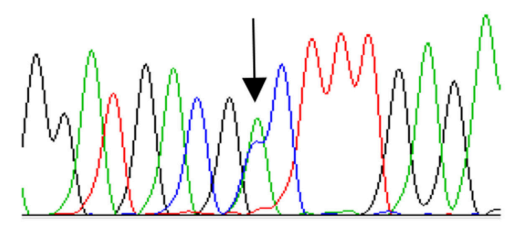

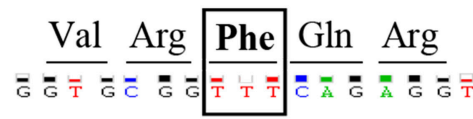

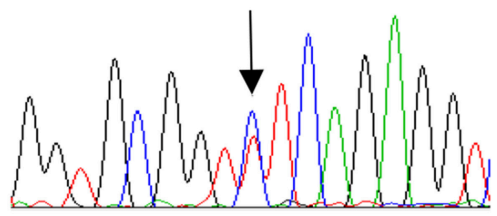

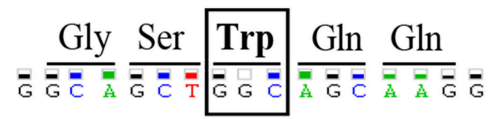

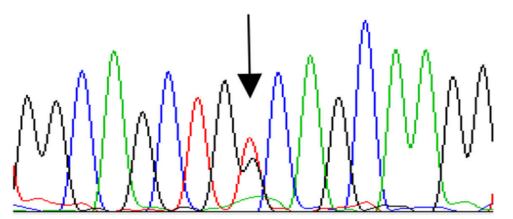

FIGURE 1 | Electropherograms showing three novel heterozygous missense variants. The arrow indicates location of the base pair substitution in: (A) MAP2K1 gene, (B) PTPN11 gene, and (C) CBL gene.

screening measures were used for the evaluation of milestones in the present study. The dysmorphology in NS also varies with ethnicity: a collaborative effort investigating NS-associated physical features in 125 individuals from diverse populations found that, the three most common physical features, present in $>70 \%$ of individuals were: widely spaced eyes $(\geq 80 \%)$, low-set ears ( $>80 \%)$ and short stature ( $>70 \%)$; ptosis was less common in black Africans (63\%), and webbed neck less common in Asians (Kruszka et al., 2017). In the present study, Black Africans were found to have the most distinctive features, with epicanthic folds, previously reported to be very common in the general black South African population (Christianson et al., 1995), being the most common feature. However, caution should be observed when interpreting the high frequency of epicanthic folds in this study, as it may be more suggestive of a common variant in the general population than a distinctive feature of NS in South African patients. Congenital heart defects represent a major cause of morbidity and mortality in affected individuals with NS of all age groups (Prendiville et al., 2014). As equally found in this study, CHD are reported in $50-80 \%$ of individuals affected with NS, the most common CHD being PS ((Hickey et al., 2011); (Prendiville et al., 2014)).

The molecular detection rate in this study was relatively low $(31.2 \%)$, compared to the expected $>70 \%$ when using whole exome sequencing (WES) or a comprehensive multigene panel testing (Aoki et al., 2016). It is unlikely that our lower detection rate could be attributed to inappropriate phenotyping, in view of the stringent patient selection process applied. This suggests that genotyping these patients using other sequencing methods such as WES may allow identification of pathogenic variants in genes not investigated in this study, particularly in the large family (Figure S1) with strong clinical features of NS and no mutation identified. All the variants detected were missense, in accordance with available data on NS (Tartaglia et al., 2002; Nava et al., 2007; Martinelli et al., 2010). Interestingly, we identified a pathogenic variant (MAP2K1 c.389A > G; p.Tyr130Cys) known to be frequently associated with Cardiofaciocutaneous syndrome (Rodriguez-Viciana et al., 2006). This patient was initially labeled with the diagnosis of NS at the age of 12 months, but the progression of clinical features later favored revision of the diagnosis to Costello syndrome. This case illustrates the challenges in clinical diagnosis of some NS patients, due to overlapping features with other RASopathies. Variability in phenotypic expression, high genetic heterogeneity and low mutation frequency in several NS genes are among the difficulties in establishing consistent correlations between known causative genes or variants and specific phenotypes. Our findings are consistent with the literature, with a positive association between short stature, PS, coagulopathy, pectus deformities of the chest, and variants in PTPN11 (Tartaglia et al., 2002; Yoshida et al., 2004). Similar to previous reports, patients with variants in MAP2K1 in our study had typical craniofacial features and skin manifestations of NS (Nava et al., 2007; Nyström et al., 2008). To date, very little is known about genotypephenotype correlations in NS patients with variants in $C B L$. In addition to presenting with less typical craniofacial features, our patient with a $C B L$ variant had a cardiovascular phenotype characterized by a combination of bicuspid aortic valve and coarctation of the aorta, which are infrequently associated with NS. 
A

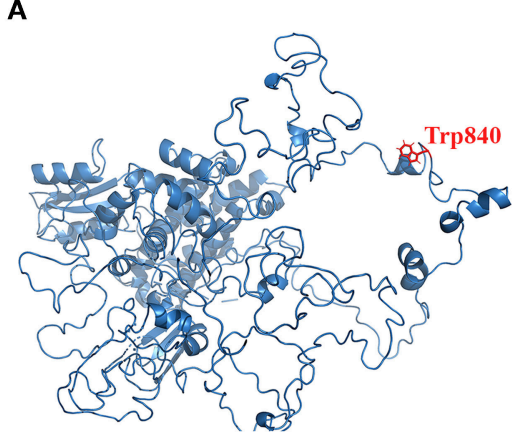

CBL

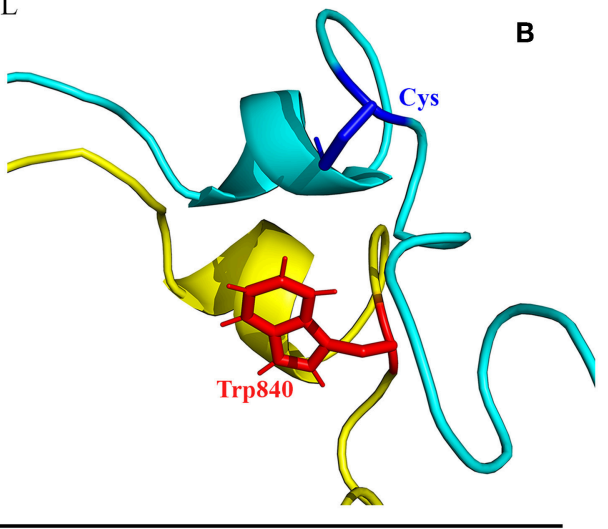

C

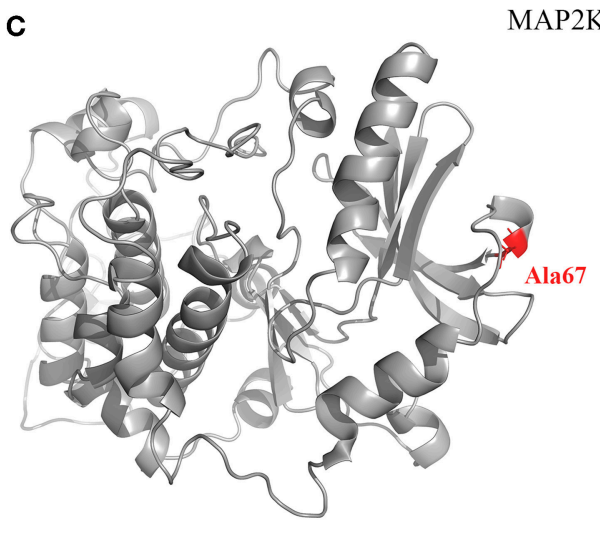

D

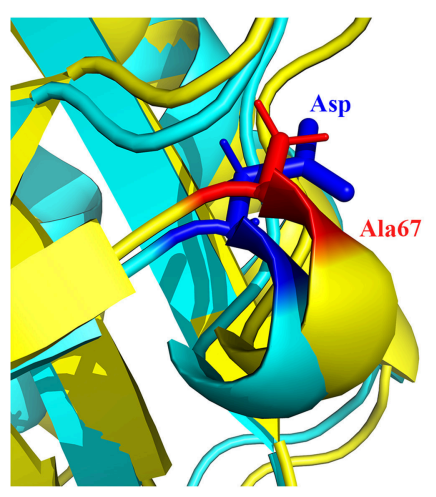

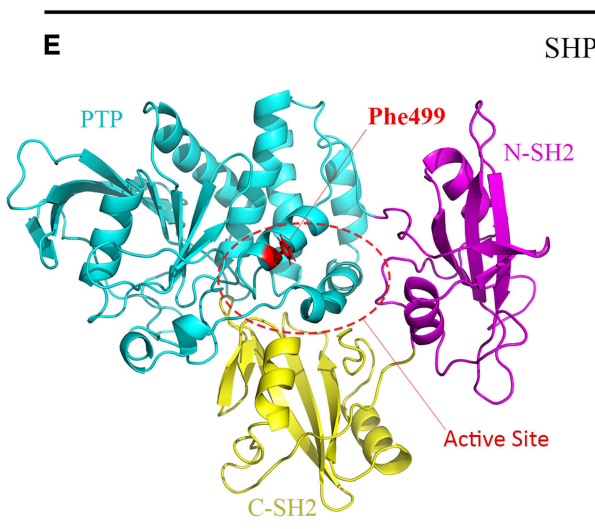

SHP-2

$\mathbf{F}$

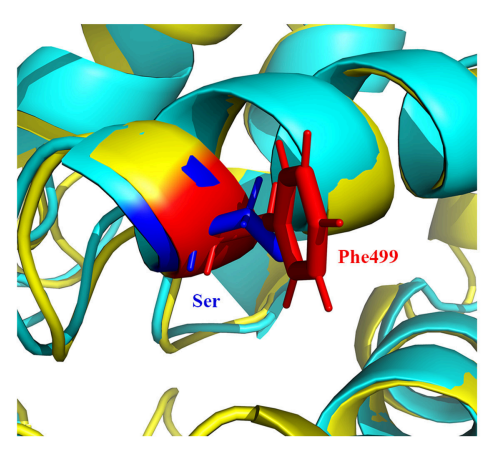

FIGURE 2 | Molecular modeling scheme of the CBL p.Cys840Trp and MAP2K1 p.Asp67Ala mutants, and crystal structure of the SHP-2 p.Ser499Phe (PDB:2SHP). (A) CBL protein with mutated residue (Trp840) colored red. The non-conservative substitution of Cys840 negatively charged and hydrophilic with Trp840 non-polar and hydrophobic may impact binding interaction, stability and the flexibility of the protein; (B) Zoom of the CBL mutation site comparing the configuration of the wild type and mutant protein and illustrating the flexibility of the structures. (C) MAP2K1 protein showing the mutated residue (Ala67) colored red. The substitution of Asp67 negatively charged and hydrophilic with Ala67 non-polar and hydrophobic potentially impact binding interactions; (D) Zoom of the MAP2K1 mutation site comparing the configuration of the wild type and mutant structures. (E) Crystal structure of SHP-2 including three domains of the protein: PTP (cyan), C-SH2 (yellow) and N-SH2 (pink). The mutated residue (Phe499) colored red is located in active site (Lee et al., 2005). The substitution of the polar and hydrophilic Ser499 with the non-polar and highly hydrophobic Phe499 in the active site of the protein potentially impact binding interactions and the stability of the new structure; (F) Zoom of the SHP-2 mutation site comparing the configuration of the wild type and mutant structures.

\section{CONCLUSION}

This first application of targeted NGS for the molecular diagnosis of NS in South Africans suggests that clinical characteristics and genotype-phenotype correlations found in affected individuals are generally similar to those reported in other populations. Therefore, careful phenotyping based on existing diagnostic criteria can effectively enable the diagnosis of most NS-affected individuals in South Africa. The use of targeted NGS in the present study have allowed for detection of novel variants in 
TABLE 4 | Summary of clinical features in mutation-positive patients.

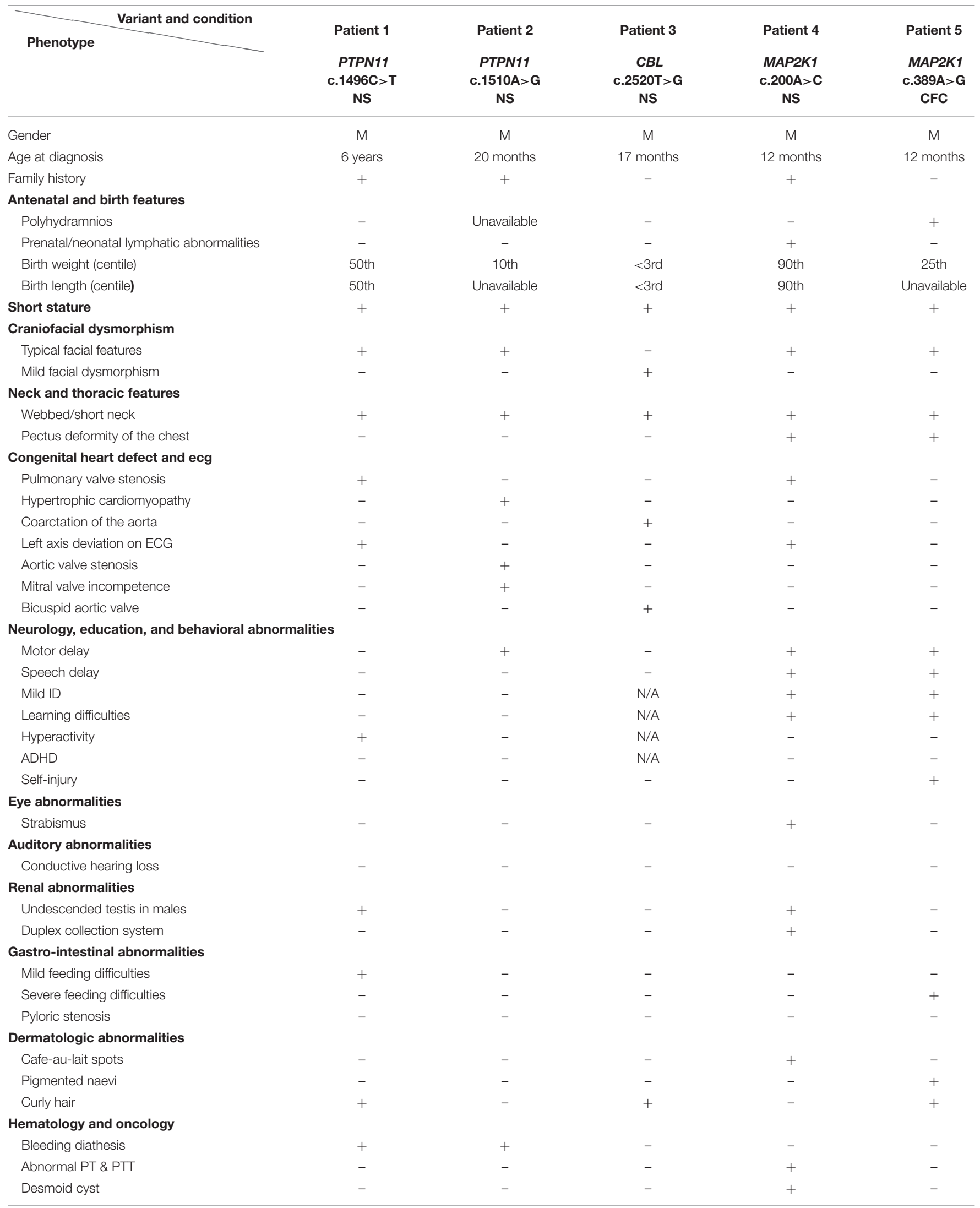

+: Feature present; -: Feature absent; N/A: not applicable. 


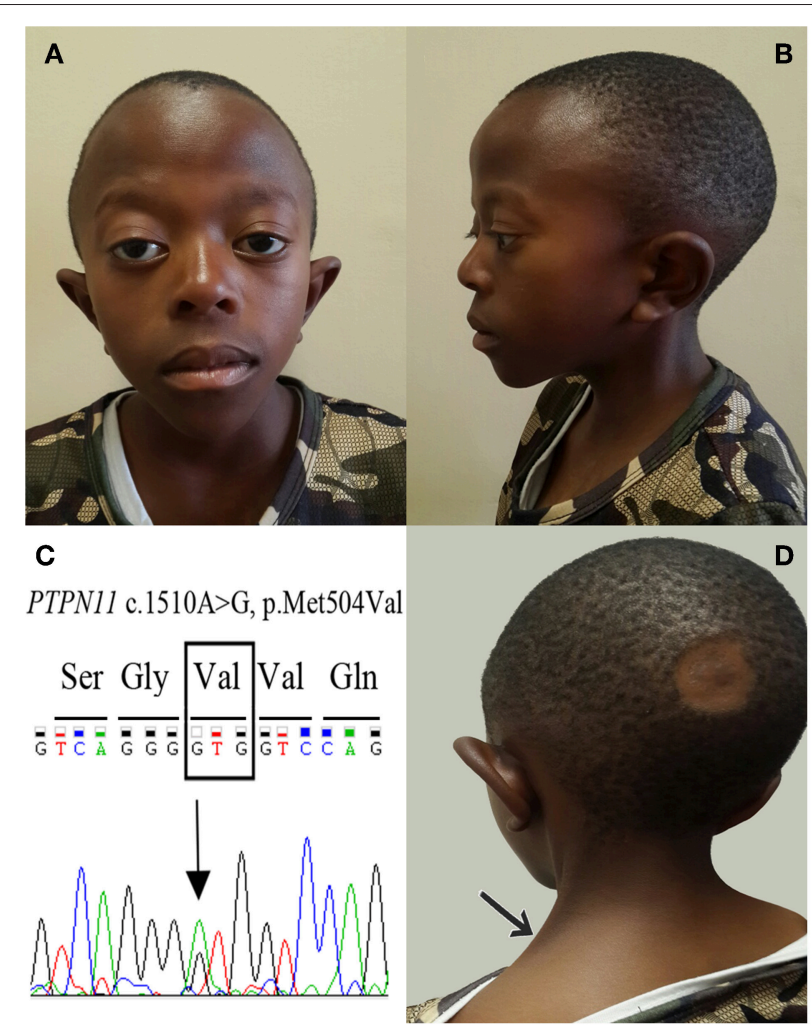

FIGURE 3 | Craniofacial features of an 11-year-old boy with NS and PTPN11 c. $1510 \mathrm{~A}>\mathrm{G}$ (p.Met504Val) variant. (A) Frontal views showing a triangular face with pointed chin; tall forehead; bilateral ptosis, predominantly on the right; sparse eyebrows; epicanthic folds and protruding ears. (B) Lateral view showing high anterior hairline with low-set and posteriorly rotated ears. (C) Electropherogram of the PTPN11 c.1510A > G (p.Met504Val) variant. (D) Posterior view with the arrow indicating webbing of the neck.

TABLE 5 | Comparisons of clinical features associated with the three genes identified.

\begin{tabular}{lccc}
\hline Characteristics & $\begin{array}{c}\boldsymbol{P} \text { PPN11 } \\
(\boldsymbol{n}=\mathbf{2})\end{array}$ & $\begin{array}{c}\mathbf{C B L} \\
(\boldsymbol{n}=\mathbf{1})\end{array}$ & $\begin{array}{c}\text { MAP2K1 } \\
(\boldsymbol{n}=\mathbf{2})\end{array}$ \\
\hline Mean age at diagnosis (year) & 3.3 & 1.4 & 1 \\
Positive family history & $2(100 \%)$ & 0 & $1(50 \%)$ \\
Antenatal features & 0 & 0 & $2(100 \%)$ \\
Short stature & $2(100 \%)$ & $100 \%$ & $2(100 \%)$ \\
Typical dysmorphic features & $2(100 \%)$ & 0 & $2(100 \%)$ \\
Webbed/short neck & $2(100 \%)$ & $100 \%$ & $2(100 \%)$ \\
Pectus deformity of the chest & 0 & 0 & $2(100 \%)$ \\
Congenital heart defects & $2(100 \%)$ & $100 \%$ & $2(100 \%)$ \\
Pulmonary valve stenosis & $1(50 \%)$ & 0 & $1(50 \%)$ \\
Hypertrophic cardiomyopathy & $1(50 \%)$ & 0 & 0 \\
Coagulopathy & $2(100 \%)$ & 0 & $1(50 \%)$ \\
Skin features & 0 & 0 & $2(100)$ \\
Intellectual disability & 0 & 0 & $2(100)$
\end{tabular}

genes infrequently associated with NS in other populations. Further studies of a larger African cohort with NS, ideally using WES, are needed.

\section{ETHICS STATEMENT}

The study was performed in accordance with the Declaration of Helsinki and with the approval of the Faculty of Health Sciences Human Research Ethics Committee, University of Cape Town (HREC: 449/2016). Written informed consent was obtained from the parents and/or the patient prior to their involvement into the study, including permission to publish photographs.

\section{AUTHOR CONTRIBUTIONS}

CT-N, AE, and AW contributed to conception and design of the study. CT-N and AW collected data; CT-N, GA, and CB performed molecular analysis and interpretation of data. CT-N wrote the first draft of the manuscript and $\mathrm{CB}$ wrote a section of the manuscript. All authors contributed to manuscript revision, read and approved the submitted version.

\section{FUNDING}

This study was supported by the South African Medical Research Council (SAMRC's) Self-initiated Research (SIR) and the Wellcome Trust/AAS Ref: H3A/18/001, to AW; NIH, USA, grant number U01HG009716 to AW. The funders had no role in study design, data collection and analysis, decision to publish, or preparation of the manuscript.

\section{ACKNOWLEDGMENTS}

The authors thank members of the clinical unit of the Division of Human Genetics, University of Cape Town for contributing to data collection. We also thank Drs. Bertram Henderson, Maureen Conradie and Sarah Kraus for their assistance in data acquisition. Finally, we are grateful to patients and families who participated in this study.

\section{SUPPLEMENTARY MATERIAL}

The Supplementary Material for this article can be found online at: https:/www.frontiersin.org/articles/10.3389/fgene. 2019.00333/full\#supplementary-material

Figure S1 | Pedigree of a family with a dominant inheritance pattern of NS, but no pathogenic variant found in the 14 genes investigated. Affected individuals (black) presented with typical craniofacial features, short stature, pectus deformities of the chest, webbed or short neck, congenital heart defect and café au lait spots. The variant CBL c.2345C>T (p.Pro782Leu) identified in the proband (IV-2), classified as uncertain significance in ClinVar, did not segregate with the disease in the family: five first or second-degree relatives were screened for the detected variant in addition to the index case, including three clinically affected and two non-affected individuals. The number beneath deceased family members indicates their age at death.

Table S1 | Summary of clinical features identified in the cohort of 26 patients. 


\section{REFERENCES}

Abdel-Salam, E., and Temtamy, S. A. (1969). Familial turner phenotype. J. Pediatr. 74, 67-72. doi: 10.1016/S0022-3476(69)80009-0

Allanson, J. E., and Roberts A. E. (2016). "Noonan Syndrome," in GeneReviews $(\mathbb{B}$ [Internet], eds M. P. Adam, H. H. Ardinger, R. A. Pagon, S. E. Wallace, L. J. H. Bean, K. Stephens, and A. Amemiya (Seattle, WA: University of Washington), 1993-2018.

Aoki, Y., Niihori, T., Inoue, S., and Matsubara, Y. (2016). Recent advances in RASopathies. J. Hum. Genet. 61, 33-39. doi: 10.1038/jhg.2015.114

Berendsen, H. J. C., Postma, J. P. M., Gunsteren, W. F. V., Dinola, A., and Haak, J. R. (1984). Molecular dynamics with coupling to an external bath molecular dynamics with coupling to an external bath. J. Chem. Phys. 81:3684. doi: $10.1063 / 1.448118$

Bussi, G., Donadio, D., and Parrinello, M. (2007). Canonical sampling through velocity rescaling canonical sampling through velocity rescaling. J. Chem. Phys. 126, 014101-014107. doi: 10.1063/1.2408420

Chen, P.-C., Yin, J., Yu, H.-W., Yuan, T., Fernandez, M., Yung, C. K., et al. (2014). Next-generation sequencing identifies rare variants associated with Noonan syndrome. PNAS. 111, 11473-11478. doi: 10.1073/pnas.1324 128111

Christianson, A. L., Kromberg, J. G., and Viljoen, E. (1995). Clinical features of Black African Neonates with Down's syndrome. East Afr. Med. J. 72, 306-310.

Cordeddu, V., Yin, J. C., Gunnarsson, C., Virtanen, C., Drunat, S., Lepri, F., et al. (2015). Activating mutations affecting the Dbl Homology domain of SOS2 cause Noonan syndrome. Hum. Mutat. 36, 1080-1087. doi: 10.1002/humu.22834

Croonen, E. A., Nillesen, W. M., Stuurman, K. E., Oudesluijs, G. L., Van De Laar, I. M. B. M., Martens, L., et al. (2013). Prenatal diagnostic testing of the Noonan syndrome genes in fetuses with abnormal ultrasound findings. Eur. J. Hum. Genet. 21, 936-942. doi: 10.1038/ejhg. 2012.285

Darden, T., York, D., and Pedersen, L. (1993). Particle mesh Ewald: an N -log (N) method for Ewald sums in large systems. J. Chem. Phys. 98, 10089-10092. doi: $10.1063 / 1.464397$

Essmann, U., Perera, L., Berkowitz, M. L., Darden, T., and Lee, H. (1995). A smooth particle mesh Ewald method. J. Chem. Phys. 103, 8577-8593. doi: $10.1063 / 1.470117$

Hess, B., Bekker, H., Berendsen, H. J. C., and Fraaije, J. G. E. M. (1997). LINCS: a linear constraint solver for molecular simulations. J. Comput. Chem. 18, 1463-1472. doi: 10.1002/(SICI)1096-987X(199709)18:12<1463::AID-JCC4>3. $0 . \mathrm{CO} ; 2-\mathrm{H}$

Hickey, E. J., Mehta, R., Elmi, M., Asoh, K., McCrindle, B. W., Williams, W. G., et al. (2011). Survival implications : hypertrophic cardiomyopathy in Noonan syndrome. Congenit. Heart Dis. 6, 41-47. doi: 10.1111/j.1747-0803.2010.00465.x

Hof, P., Pluskey, S., Dhe-paganon, S., Eck, M. J., and Shoelson, S. E. (1998). Crystal structure of the tyrosine phosphatase SHP-2. Cell 92, 441-450. doi: 10.1016/S0092-8674(00)80938-1

Johnston, J. J., Van der Smagt, J. J., Rosenfeld, J. A., Pagnamenta, A. T., Alswaid, A., and Baker, E. H. (2018). Autosomal recessive Noonan syndrome associated with biallelic LZTR1 variants. Genet. Med. 20, 1175-1185. doi: 10.1038/gim.2017.249

Jorgensen, W. L., and Tirado-rives, J. (1988). The OPLS potential functions for proteins. Energy minimizations for crystals of cyclic peptides and crambin. J. Am. Chem. Soc. 110, 1657-1666. doi: 10.1021/ja00214a001

Koboldt, D. C., Zhang, Q., Larson, D. E., Shen, D., McLellan, M. D., Lin, L., et al. (2012). VarScan 2: somatic mutation and copy number alteration discovery in cancer by exome sequencing. Genome Res. 22, 568-576. doi: $10.1101 /$ gr.129684.111

Kruszka, P., Porras, A. R., Addissie, Y. A., Moresco, A., Medrano, S., Mok, G. T. K., et al. (2017). Noonan syndrome in diverse populations. Am. J. Med. Genet. A. 173, 1-12. doi: 10.1002/ajmg.a.38362

Lebeko, K., Manyisa, N., Chimusa, E. R., Mulder, N., Dandara, C., and Wonkam, A. (2017). A genomic and protein - protein interaction analyses of nonsyndromic hearing impairment in Cameroon using targeted genomic enrichment. OMICS. 21, 1-10. doi: 10.1089/omi.2016.0171
Lee, W. H., Raas-rotschild, A., Miteva, M. A., Bolasco, G., Rein, A., Gillis, D., et al. (2005). Noonan syndrome type I with PTPN11 3 bp deletion: structure function implications. Proteins 58, 7-13. doi: 10.1002/prot.20296

Li, H., Handsaker, B., Wysoker, A., Fennell, T., Ruan, J., Homer, N., et al. (2009). The sequence alignment/map format and SAMtools. Bioinformatics 25, 20782079. doi: 10.1093/bioinformatics/btp352

Martinelli, S., De Luca, A., Stellacci, E., Rossi, C., Checquolo, S., Lepri, F., et al. (2010). Heterozygous germline mutations in the $C B L$ tumor-suppressor gene cause a Noonan syndrome-like phenotype. Am. J. Hum. Genet. 87, 250-257. doi: 10.1016/j.ajhg.2010.06.015

Maximilian, C., Ioan, D., and Fryns, J. (1992). A syndrome of mental retardation, short stature, craniofacial anomalies with palpebral ptosis and pulmonary stenosis in three siblings with normal parents. An example of autosomal recessive inheritance of the Noonan phenotype?. Genet. Couns. 3, 115-118.

Mckenna, A., Hanna, M., Banks, E., Sivachenko, A., Cibulskis, K., Kernytsky, A., et al. (2010). The Genome Analysis Toolkit : A MapReduce framework for analyzing next-generation DNA sequencing data. Genome Res. 20, 1297-1303. doi: $10.1101 /$ gr.107524.110

Mendez, H. M., and Opitz, J. M. (1985). Noonan syndrome: a review. Am. J. Med. Genet. 21, 493-506. doi: 10.1002/ajmg.1320210312

Nakaguma, M., Jorge, A. A. L., and Arnhold, I. J. P. (2019). Noonan syndrome associated with growth hormone deficiency with biallelic LZTR1 variants. Genet. Med. 21:260. doi: 10.1038/s41436-018-0041-5

Nava, C., Hanna, N., Michot, C., Pereira, S., Pouvreau, N., Niihori, T., et al. (2007). Cardio-facio-cutaneous and Noonan syndromes due to mutations in the RAS/MAPK signalling pathway: genotype- phenotype relationships and overlap with Costello syndrome. J. Med. Genet. 44, 763-771. doi: 10.1136/jmg.2007.050450

Neira, J., Horn, J., Tardos, J. D., and Schmidt, G. (1996). "Multisensor mobile robot localization," in International Conference on Robotics and Automation, Proceedings of the IEEE (Minneapolis, MN), 673-679.

Nelen, M., Costa, J. L., Neveling, K., and Tartaglia, M. (2014). "Development and verification of a Noonan genes Ion AmpliSeq ${ }^{\mathrm{TM}}$ panel," in American Society of Human Genetics Annual Meeting 2014 (San Diego, CA).

Nyström, A.-M., Ekvall, S., Berglund, E., Björkqvist, M., Braathen, G., Duchen, K., et al. (2008). Noonan and cardio-facio-cutaneous syndromes: two clinically and genetically overlapping disorders. J. Med. Genet. 45, 500-506. doi: $10.1136 /$ jmg.2008.057653

Pierpont, E. I. (2016). Neuropsychological functioning in individuals with noonan syndrome : a systematic literature review with educational and treatment recommendations. J. Pediatr. Psychol. 2, 14-33. doi: 10.1007/s40817-015-0005-5

Prendiville, T. W., Gauvreau, K., Tworog-Dube, E., Patkin, L., Kucherlapati, R. S., Roberts, A. E., et al. (2014). Cardiovascular disease in Noonan syndrome. Arch. Dis. Child. 99, 629-634. doi: 10.1136/archdischild-2013-305047

Pronk, S., Apostolov, R., Shirts, M. R., Smith, J. C., Kasson, P. M., Spoel, D. V. D., et al. (2013). GROMACS 4.5: a high-throughput and highly parallel open source molecular simulation toolkit'. Bioinformatics 29, 845-854. doi: 10.1093/bioinformatics/btt055

Roberts, A. E., Allanson, J. E., Tartaglia, M., and Gelb, B. D. (2013). Noonan syndrome. Lancet 381, 333-342. doi: 10.1016/S0140-6736(12)61023-X

Roberts, A. E., Araki, T., Swanson, K. D., Montgomery, K. T., Schiripo, T. A., Joshi, V. A., et al. (2007). Germline gain-of-function mutations in SOS1 cause Noonan syndrome. Nat. Genet. 39, 70-74. doi: 10.1038/ng1926

Rodriguez-Viciana, P., Tetsu, O., Tidyman, W. E., Estep, A. L., Conger, B. A., Cruz, M. S., et al. (2006). Germline mutations in genes within the MAPK pathway cause cardio-facio-cutaneous syndrome. Science 311, 1287-1291. doi: $10.1126 /$ science. 1124642

Romano, A. A., Allanson, J. E., Dahlgren, J., Gelb, B. D., Hall, B., Pierpont, M. E., et al. (2010). Noonan syndrome: clinical features, diagnosis, and management guidelines. Pediatrics 126, 746-759. doi: 10.1542/peds.2009-3207

Sharland, M., Burch, M., McKenna, W. M., and Paton, M. A. (1992). A clinical study of Noonan syndrome. Arch. Dis. Child. 67, 178-183. doi: 10.1136/adc.67.2.178

Tartaglia, M., and Gelb, B. D. (2005). Noonan syndrome and related disorders: genetics and pathogenesis. Annu. Rev. Genomics Hum. Genet. 6, 45-68. doi: 10.1146/annurev.genom.6.080604.162305 
Tartaglia, M., Kalidas, K., Shaw, A., Song, X., Musat, D. L., Van der Burgt, I., et al. (2002). PTPN11 Mutations in Noonan syndrome: molecular spectrum, genotype-phenotype correlation, and phenotypic heterogeneity. Am. J. Hum. Genet. 70, 1555-1563. doi: 10.1086/340847

Tekendo-Ngongang, C., Dahoun, S., Nguefack, S., Gimelli, S., Sloan-Béna, F., and Wonkam, A. (2014). Challenges in clinical diagnosis of WilliamsBeuren syndrome in sub-Saharan Africans: case reports from Cameroon. Mol. Syndromol. 5, 287-292. doi: 10.1159/000369421

Van der Burgt, I. (2007). Noonan syndrome. Orphanet J. Rare Dis. 2:4. doi: 10.1186/1750-1172-2-4

Van Der Burgt, I., and Brunner, H. (2000). Genetic heterogeneity in Noonan syndrome: evidence for an autosomal recessive form. Am. J. Med. Genet. 94, 46-51. doi: 10.1002/1096-8628(20000904)94:1<46::AID-AJMG10>3.0.CO;2-I

Wang, K., Li, M., and Hakonarson, H. (2010). ANNOVAR : functional annotation of genetic variants from high-throughput sequencing data. Nucleic Acids Res. 38, 1-7. doi: $10.1093 / \mathrm{nar} / \mathrm{gkq} 603$

Yamamoto, G. L., Aguena, M., Gos, M., Hung, C., Pilch, J., Fahiminiya, S., et al. (2015). Rare variants in SOS2 and LZTR1 are associated with Noonan syndrome. J. Med. Genet. 52, 413-421. doi: 10.1136/jmedgenet-2015-103018
Yoshida, R., Hasegawa, T., Hasegawa, Y., Nagai, T., Kinoshita, E., Tanaka, Y., et al. (2004). Protein-tyrosine phosphatase, nonreceptor type 11 mutation analysis and clinical assessment in 45 patients with Noonan syndrome. J. Clin. Endocrinol. Metab. 89, 3359-3364. doi: 10.1210/jc.2003032091

Zhang, Y. (2008). I-TASSER server for protein 3D structure prediction. BMC Bioinformatics 9, 1-8. doi: 10.1186/1471-2105-9-40

Conflict of Interest Statement: The authors declare that the research was conducted in the absence of any commercial or financial relationships that could be construed as a potential conflict of interest.

Copyright $\odot 2019$ Tekendo-Ngongang, Agenbag, Bope, Esterhuizen and Wonkam. This is an open-access article distributed under the terms of the Creative Commons Attribution License (CC BY). The use, distribution or reproduction in other forums is permitted, provided the original author(s) and the copyright owner(s) are credited and that the original publication in this journal is cited, in accordance with accepted academic practice. No use, distribution or reproduction is permitted which does not comply with these terms. 